\title{
LA ENSEÑANZA DE LAS CIENCIAS BÁSICAS EN MEDICINA DESDE LA PERSPECTIVA DE LA JUSTIFICACIÓN EPISTEMOLÓGICA DEL CURRÍCULO
}

\author{
Jorge Granados Zúñiga \\ Docente de la Escuela de Medicina de la Universidad de Costa Rica \\ San José, Costa Rica
}

Recibido 15-V-2009 • Aceptado 22-X-2009 • Corregido 11-XI-2009

\begin{abstract}
Resumen: Un elemento fundamental en el diseño curricular es el epistemológico. En algunos de los cursos de ciencias básicas (Bioquímica y Fisiología) de la carrera de Medicina de la Universidad de Costa Rica, he notado la ausencia de coherencia epistemológica entre el conocimiento que la ciencia produce y aquel que es interiorizado por el estudiante. Esto obligaría a realizar una evaluación del currículo en cuya labor los docentes tienen una perspectiva ventajosa porque son los que mejor conocen el tipo de estudiante que sigue la carrera. Cuando el docente se enfrenta con preguntas que cuestionan los limites entre posiciones epistemológicas diversas, se ve confrontado con la disyuntiva de proponer explicaciones que van de lo antropomórfico a lo causal. Aunque en la enseñanza de ciencias básicas en la carrera de Medicina de la Universidad de Costa Rica no parece estar permeando explícitamente una fundamentación epistemológica claramente definida, en ocasiones podrian notarse rasgos positivistas, empiristas y fenoménicos, con elementos de una filosofía determinista y métodos de evaluación cuantitativos. Dadas las ventajas y desventajas que ofrecen las diferentes propuestas epistemológicas propongo la necesidad de una definición más clara de la justificación epistemológica curricular de los cursos de ciencias básicas de la carrera Medicina $y$, eventualmente, de la carrera misma.
\end{abstract}

Palabras clave: Epistemología, currículo, ciencias básicas, Medicina.

\section{Introducción:}

\section{Necesidad de la justificación epistemológica del currículo}

De acuerdo con Quesada, Cedeño y Zamora (2001), la fundamentación de un currículo especifica el marco epistemológico de la carrera, esto es, define el objeto de estudio, la finalidad del conocimiento, la relación con otras disciplinas, las áreas disciplinarias, los ejes curriculares y permite identificar el paradigma metodológico y los principios pedagógicos para los procesos de enseñanza y aprendizaje.

Para Díaz-Barriga, Lule, Pacheco, Saad y Rojas-Drummond (1997), el nivel epistemológico en el diseño curricular se refiere a la forma en que se concibe el problema del conocimiento. Debe considerarse la forma en que los estudiantes universitarios van adquiriendo, construyendo y transformando el conocimiento de acuerdo con la etapa de desarrollo cognoscitivo en la que se encuentran.

Sin embargo, de acuerdo con estos autores, no existe una continuidad entre el conocimiento que la ciencia produce y 


\begin{abstract}
A fundamental element in the curricular design is the epistemological one. In some courses of Basic Sciences (Biochemistry and Physiology) in the Medicine career at the University of Costa Rica there is not an evident epistemological coherence between the knowledge that science produces and the one that is assimilated by the student. This would lead to make a curricular evaluation in which professors have an advantageous perspective because they are the ones who know the type student who sign up in the career. When a professor faces questions that challenge the limits between different epistemological positions, he or she is confronted with the dilemma of proposing explanations going from the anthropomorphic to the causal. Although the teaching of Basic Sciences in the career of Medicine of the University of Costa Rica does not seem to be explicitly permeating a clearly defined epistemological rationale, positivist traits can often be noticed, as well as empirical and phenomenological characteristics with deterministic philosophy elements and quantitative methods of evaluation. Given the advantages and disadvantages of different epistemological approaches, it is proposed that a clear definition of the curricular epistemological justification is essential to the Basic Science courses of Medicine and of the career itself.
\end{abstract}

Key words: Epistemology, curriculum, basic sciences, Medicine. aquel que es interiorizado por el estudiante, pues en todos los niveles de la construcción curricular existen filtros que operan en la selección, construcción y validación del conocimiento, y esto tiene que ver con las representaciones de lo real que poseen los individuos que participan en la práctica educativa, incluidos los docentes y estudiantes. En este sentido, una clara justificación epistemológica del currículo y de los cursos es necesaria para reducir el efecto que estos "filtros" producen sobre la continuidad entre el conocimiento que la ciencia produce y el que es interiorizado por el estudiante. Para ello, habría que procurar una coherencia epistemológica entre "explicaciones de una disciplina" y "explicaciones instruccionales", según las define Talanquer (2007).

Idealmente, esta coherencia epistemológica debería establecerse al momento de creación de la carrera pero también puede ser que, según lo advierte Roldán (2005), un plan de estudio ya vigente requiera replantear las teorías de aprendizaje de manera que los cursos tengan contenidos seleccionados para lograr la construcción del conocimiento a partir de un enfoque epistemológico específico coherente con la formación integral del individuo. En este sentido, Hernández (1999) también señala que la comprensión de la práctica docente necesita del estudio de las disciplinas desde una perspectiva epistemológica. Por esta razón, el docente, como uno de los elementos fundamentales en los procesos de enseñanza y aprendizaje y como conocedor directo de las dificultades metodológicas y conceptuales que esto implica, debería ser uno de los primeros promotores y protagonistas del proceso de revisión e innovación curricular (ver la sección 2 del presente documento: Papel del docente en la revisión curricular).

En el programa de estudios de la carrera de Medicina de la Universidad de Costa Rica después de los Estudios Generales vienen dos ciclos lectivos que "integran las materias básicas clínicas" (Universidad de Costa Rica. Escuela de 
Medicina, 2005, p. 10). Estos cursos de ciencias básicas se imparten en las aulas y laboratorios del edificio de la Escuela de Medicina y son el fundamento del resto de la carrera. Entre estos cursos se encuentran Fisiología, Bioquímica, Anatomía y Farmacología. Mi experiencia docente se ha desarrollado en torno a los cursos de Bioquímica y Fisiología y, a partir de dicha experiencia, se me ha hecho cada vez más evidente la importancia de la discusión en torno a la forma en que se concibe el problema de la construcción del conocimiento en la carrera de Medicina. Esta discusión resulta especialmente relevante con miras a definir con claridad un paradigma metodológico que permita una mayor coherencia epistemológica entre las explicaciones que aportan las ciencias básicas (Bioquímica y Fisiología, en el caso particular que aquí me interesa) y las explicaciones instruccionales que forman parte del cuerpo de conocimiento de la carrera de Medicina que se imparte a los estudiantes.

A pesar de la relevancia que tiene contar con una clara justificación epistemológica de la carrera de Medicina que permita orientar la práctica docente en cada uno de los cursos en particular, en la actualidad tal fundamentación aún no existe, según trato de detallar en la sección 3: Fundamento epistemológico de las ciencias básicas en la carrera de Medicina en la actualidad. Por esta razón, en la sección 4: Propuesta epistemológica para la enseñanza de las ciencias básicas en la carrera de Medicina, intento sugerir algunos elementos iniciales que podrían tenerse en cuenta al momento de abordar este importante problema en la enseñanza de la Medicina en nuestra Universidad.

\section{Papel del docente en la revisión curricular}

Para Roldán (2005), en la evaluación de un currículo deben participan los docentes responsables de interactuar con los estudiantes en el aula ya que, según dicho autor, son ellos los que mejor conocen el tipo de estudiante que sigue la carrera y las necesidades de los cursos que corresponden al plan de estudios.

Se ha propuesto la teoría crítica y cooperativa del currículo (Hernández, 1999) la cual señala que las partes implicadas en el proceso de enseñanza asumen una actitud constructiva y renovadora. Así, se sugiere que los educadores inicien con el estudio de algunas contradicciones que pueden expresarse en el aula para luego identificar las maneras de mejorar el currículo. En mi práctica docente en particular he percibido que una aparente contradicción puede surgir ante preguntas que podrían ser abordadas desde posiciones epistemológicas alternativas; por ejemplo, preguntas del tipo "¿cómo ocurre la regulación hormonal de la glucosa sanguínea?” o “ipor qué ocurre la regulación hormonal de la glucosa sanguínea?". En los cursos de Bioquímica y Fisiología para Medicina, la respuesta a estas preguntas, u otras similares, puede conducir a explicaciones causales (el primer tipo de pregunta planteado) o a explicaciones antropomórficas (el segundo tipo de pregunta) y, dentro de estas últimas, comúnmente pueden surgir los razonamientos teleológicos (según Ferrater Mora, 2007, el término "teleológico" se refiere a un modo de explicación basado en causas finales, a diferencia del modo de explicación basado en causas eficientes). Por ejemplo, siguiendo con el fenómeno citado anteriormente, puede sugerirse una explicación basada en mecanismos de señalización neuro-endocrina o puede pensarse en que el organismo regula la glucosa sanguínea porque busca la estabilidad de este parámetro.

En este punto surge una función crítica del docente: convertir las explicaciones de una disciplina específica en explicaciones instruccionales dentro de un cuerpo de información coherente con un diseño curricular determinado. Esta se define como una "transposición didáctica" en el sentido de que implica reelaborar una información desde su fuente primaria para presentarla 
de una manera coherente y comprensible a los estudiantes. Al abordar dicha "transposición" el docente se enfrenta con la posibilidad de asumir diversas perspectivas epistemológicas alternativas y es importante que pueda definir cuáles son las ventajas y desventajas de las mismas. Por ejemplo, según Richardson (1990), las funciones corporales pueden pensarse en términos de su propósito teleológico (¿por qué ocurren?) o de sus mecanismos de acción (¿cómo ocurren?). Aunque la Fisiología y la Bioquímica, al ser disciplinas científicas, se ocupan del segundo abordaje, este autor sugiere que alguna consideración teleológica puede ser beneficiosa para orientar al estudiante hacia el papel integrativo $u$ homeostático de una función particular llevada a cabo por un tejido, órgano o sistema de órganos. Una explicación teleológica, aunque subjetiva por parte del instructor, puede ayudarle al estudiante para comprender los mecanismos por los cuales se realizan las funciones corporales. Sin embargo, como es la operación de los mecanismos la que determina si una función se realiza, no la necesidad de la función por sí misma, es importante que al considerar las funciones corporales, los estudiantes no confundan el propósito teleológico con los mecanismos de acción. Este tipo de consideraciones epistemológicas usualmente no se han tenido en cuenta al pensar en la justificación de la carrera y de los cursos pero deberían incorporarse en el currículo, según se propone más adelante.

El docente de Biología, al momento de reflexionar sobre la orientación epistemológica de la enseñanza en este campo del conocimiento debería tomar en cuenta dos elementos explicativos que subyacen en la gran mayoría de los fenómenos biológicos: por una parte la teoría de la selección natural y, por otra parte, los mecanismos de conservación y expresión de la información genética. Con respecto al primero, para Zohar y Ginossar (1998) la selección natural es la fuerza direccional cuyo fin es la adaptabilidad y el científico que trabaja en este campo, o el docente que la enseña, no debe confundir la pregunta ¿por qué? con la pregunta ¿con qué propósito? Para evitar esta confusión debe distinguirse entre las explicaciones funcionales y las explicaciones evolutivas. En este caso, la explicación funcional responde al ¿cómo?, la explicación evolutiva responde al ¿por qué? Estrechamente relacionado con lo anterior está el hecho de que la Biología Funcional también maneja varios aspectos de la decodificación de la información genética y una tendencia natural a la constante mejoría del código genético; esta mejoría genética es la adaptación evolutiva controlada por la selección natural. Ambos sistemas, genético y evolutivo, que parecen orientados a un fin, no contradicen la causalidad mecánica: el código genético es el plan para las actividades y la conducta del organismo (Zohar y Ginossar, 1998). Es papel del docente de ciencias biológicas aclarar los alcances explicativos de estas teorías y delimitar sus implicaciones.

\section{Fundamento epistemológico de las ciencias básicas en la carrera de Medicina en la actualidad}

En la enseñanza de las ciencias básicas para Medicina no siempre hay una distinción clara entre los abordajes causales-mecanísticos y los antropomórficosteleológicos. Para Talanquer (2007) existe una distinción entre explicaciones de una disciplina y explicaciones instruccionales: las primeras pretenden dilucidar y predecir fenómenos, se asocian con teorías y evidencias y se organizan por medio de ciertas convenciones retóricas dentro de un determinado dominio, mientras que las segundas se diseñan para enseñar, para comunicar un aspecto particular del conocimiento acerca del tema. Estas últimas son contribuciones que los profesores y los libros de texto hacen a los procesos de aprendizaje y son herramientas poderosas para ayudar a los estudiantes a entender conceptos, ideas, eventos y procedimientos. 
En general, las explicaciones causales en una disciplina científica implican la especificación de un grupo de condiciones antecedentes que son necesarias para que el evento ocurra y un conjunto de leyes generales que determinan la conducta del sistema (Talanquer, 2007). Pero, al elaborar explicaciones instruccionales, las respuestas a “Por qué?” algunas veces se construyen en términos de las consecuencias de un evento más que en términos de sus antecedentes. Esto da origen a explicaciones teleológicas cuyo poder explicativo ha sido objeto de un largo debate filosófico: por una parte, no se puede negar que las explicaciones teleológicas juegan un papel importante en la comprensión de fenómenos complejos o que se abordan por primera vez. Así, por ejemplo, para Piaget (1967), en el niño nace la idea de que todas las cosas están dirigidas hacia un fin y, de aquí, resultaría natural que incorpore las explicaciones del mundo que le rodea en términos "finalistas". Sin embargo, cuando se trata de explicaciones de fenómenos naturales formuladas en un estudiante universitario puede ocurrir la confusión de propósitos con causas si se atribuyen erróneamente características mentales humanas a objetos no humanos (antropomorfismo) (Talanquer). Aunque muchos filósofos involucrados en estas discusiones han construido fuertes argumentos a favor de la legitimidad, poder explicativo y penetración de las explicaciones teleológicas, este tipo de explicaciones generalmente se toman con suspicacia por parte de los científicos. En las ciencias naturales la noción de propósito se ha visto como superflua y se asume que cada explicación teleológica puede, eventualmente, reducirse a una explicación causal. La controversia alrededor del uso de explicaciones teleológicas en las ciencias naturales ocurrió primeramente en el domino biológico en el que términos como "función", "papel” y "propósito" son usados comúnmente para describir la conducta de organismos vivos.

Talanquer (2007) ahonda más en el tema del arraigo y validez de las explicaciones teleológicas al señalar que éstas, aunque frecuentemente se perciben como antropomórficas (los fenómenos irían dirigidos hacia una meta definida por un ser consciente), también pueden serlo solamente en un sentido metafórico. En este sentido metafórico de la explicación teleológica no está implicado el requerimiento de que los objetos o procesos conscientemente tiendan hacia una meta específica.

Apoyando la validez de las explicaciones finalistas en las ciencias biológicas, Zohar y Ginossar (1998) señalan que tales razonamientos prevalecen en Biología porque la estructura física de los organismos vivos usualmente está adaptada a su sobrevivencia y, debido a ello, parecen estar orientados hacia un fin. Al asumir una explicación teleológica se gana en valor explicativo aparente ya que se hace más accesible la comprensión de diversos fenómenos que, de otro modo, resultarían poco familiares y afines para la persona que los aborda por primera vez. Además, existe un valor heurístico en el abordaje teleológico ya que permite convertir una larga formulación en una más breve creando empatía hacia los temas científicos. Sin embargo, a pesar de estas posibles ventajas, existe el gran riesgo de atribuirle una dirección divina a una causalidad teleológica y, con ello, de que surja la contradicción entre tales explicaciones las explicaciones causales científicas.

Tamir y Zohar (1991) señalan que las explicaciones teleológicas parecen implicar que el beneficio derivado de una estructura o proceso particulares es explicación suficiente y que no hay necesidad de buscar un mecanismo que se refiera a ellas. La aceptación de las explicaciones teleológicas implica la atribución de conciencia a los seres no humanos o incluso a los diferentes órganos y esto conduce al error. Los autores mencionados también señalan que se ha realizado una distinción entre las formulaciones y las explicaciones: las primeras se pueden adquirir con relativa facilidad, las segundas son más complejas y requieren tratamiento en profundidad y 
extensivo. La teleología puede considerarse como un caso especial de antropomorfismo pues estas explicaciones hacen sentir a quien las formula que realmente entiende un fenómeno debido a que la explicación se da en términos de propósitos e intensiones que se ajustan con la forma en que él o ella acostumbra ver su propia conducta. Por ejemplo, los estudiantes creen comprender mejor la relación entre insulina y glicemia cuando suponen que la primera pretende o busca reducir la segunda. Los autores previamente indicados señalan que no es tan simple evitar el uso de formulaciones teleológicas. Por una parte, parece que es válido y real el peligro de confusión con respecto a la naturaleza de la causa y el efecto, pero, por otra parte, los científicos muchas veces usan formulaciones teleológicas para simplificar la comunicación entre ellos; ejemplos de estas explicaciones aparecidas en recientes publicaciones científicas es la relación entre el consumo de yodo y la producción de hormonas tiroideas (Yun, Lee, Bazar, Daniel y Doux, 2005), el valor adaptativo de la anorexia durante las infecciones bacterianas (Bazar, Yun y Lee, 2005) y el uso de codones sinónimos (Pluhar, 2006).

En concordancia con este razonamiento, también se ha demostrado que el abordaje teleológico tiene valor heurístico real (Tamir y Zohar, 1991) y se han desarrollado algunas ideas muy importantes como la complementariedad entre estructura y función, la adaptación de los organismos a su ambiente y la homeostasis. La experiencia educativa ha demostrado que la teleología tiene un valor heurístico pedagógico que ayuda a los estudiantes a organizar hechos y comprender mejor los fenómenos y procesos naturales.

A partir de las definiciones sobre epistemología y práctica docente sugeridas por Salas (2002) y según mi experiencia particular en el ejercicio docente, no se ha definidoexplícitamenteunafundamentación epistemológica claramente señalada en la carrera de Medicina de la Universidad de
Costa Rica. Lo anterior también se desprende de la documentación existente relacionada con los procesos de reacreditación de dicha carrera (por ejemplo, Universidad de Costa Rica. Escuela de Medicina, 2005). Habitualmente surgen rasgos positivistas, empiristas y fenoménicos, con elementos de una filosofía determinista y métodos de evaluación cuantitativos. Esta epistemología, según Salas (2002), tendría la desventaja de que reduce la posibilidad de encontrar "el sentido" de un fenómeno y de evaluarlo en su complejidad y multicausalidad. En este sentido, cabe agregar que en las ciencias naturales no se pueden obviar las implicaciones éticas y estéticas ni las consecuencias sociales que tienen los hallazgos científicos ya que son producidos en cierto contexto por seres humanos y para ser puestas en escena a nivel social.

Con base en lo expuesto hasta ahora cabe señalar dos elementos fundamentales: 1. no existe una fundamentación epistemológica definida en la carrera de Medicina de la Universidad de Costa Rica, y 2. la práctica docente podría estar reflejando un orientación epistemológica insuficiente en la que otros elementos gnoseológicos podrían mejorar la calidad de la enseñanza siempre y cuando se identifiquen claramente y se señalen sus alcances y limitaciones. Por esta razón, justifico señalar algunos planteamientos que podrían facilitar el abordaje de este importante problema en la enseñanza de la Medicina en nuestra Universidad.

\section{Propuesta epistemológica para la enseñanza de las ciencias básicas en la carrera de Medicina}

Según Modell (2007), dentro de los principales problemas que se presentan en la enseñanza de la Fisiología están que para la mayoría de los estudiantes el aprendizaje es simplemente la adquisición irreflexiva de 
información, que la mayoría de los textos son descriptivos en vez de enfocarse en la relación causal que hay entre los fenómenos, y que los estudiantes deben examinar fenómenos a diferentes niveles de complejidad y desde diferentes puntos de vista, lo que les demanda un esfuerzo de integración de información para el que no están preparados. A lo anterior habría que agregar, de acuerdo con mi experiencia particular, la poca distinción epistemológica que existe entre los abordajes causales-mecanísticos y antropomórficos-teleológicos para la enseñanza de las ciencias básicas, según se indicó en la sección precedente.

Otros elementos que deben tenerse en cuenta al plantear una propuesta epistemológica para la enseñanza de las ciencias básicas son: la naturaleza de la disciplina que se enseña, las formas en que se enseña y cuál es el aporte que se espera de los estudiantes para aprender tal disciplina (Michael, 2006b). En esta línea de pensamiento, si la Fisiología y la Bioquímica son difíciles porque requieren de la aplicación de razonamiento causal, entonces primero se debe modelar este tipo de razonamiento en los estudiantes y darles amplias oportunidades de practicarlo al tiempo que reciben una retroalimentación apropiada. Se hace necesario cuestionarse qué es lo que necesitan saber y hacer los estudiantes cuando completen el o los cursos. La respuesta a esta pregunta y otras similares puede obtenerse investigando en los requerimientos de los cursos subsecuentes en el plan de estudio de la carrera (Michael, 2006b). Una vez definido lo anterior se puede facilitar la clarificación de los alcances y limitaciones que presentan las explicaciones causales-mecanísticas en comparación con las explicaciones antropomórficas-teleológicas para poder sacar provecho de lo mejor de cada una de ellas.

Así, de acuerdo con lo que señalado hasta ahora, el problema fundamental de plantear una propuesta epistemológica para la enseñanza de las ciencias básicas en la carrera de Medicina podría girar en torno a decidir un abordaje explicativo causal-mecanístico o uno antropomórficoteleológico, o bien una combinación de ambas alternativas siempre y cuando se delimiten claramente los alcances y condiciones de cada una de ellas.

En la búsqueda de una mejor definición de las posibilidades explicativas de los dos abordajes previamente indicados cabe señalar que, según Michael (2006b), los educadores de Biología tienden a prohibir el uso de formulaciones antropomórficasteleológicas en los libros de texto y en las aulas, aunque tales formulaciones no induzcan a ningún daño real sino que, muy por el contrario, podrían significar un beneficio pedagógico potencial. Según este autor, el funcionalismo (es decir, las explicaciones causales-mecanísticas) no puede cubrir el rango completo de las explicaciones teleológicas en Biología. Por ejemplo, se puede describir con bastante precisión la secuencia de mecanismos que ocurre desde la ingesta de alimentos: el aumento de la glicemia, la liberación de insulina y la recuperación de la glicemia basal. Sin embargo, con una explicación de este tipo no se entiende por qué la glicemia normal en ayuno es de 70 a 100 $\mathrm{mg} / \mathrm{dl}$ y no otra.

De esta manera, aunque apropiado para describir los fenómenos actuales, el funcionalismo por sí solo no puede explicar las causas históricas que formaron las características biológicas y el surgimiento de los procesos tal y como se conocen en la actualidad (Zohar y Ginossar, 1998). Se ha señalado que el uso de formulaciones antropomórficas-teleológicas (entre comillas y con la advertencia explícita) puede tener un valor pedagógico real y que no necesariamente implican un riesgo para el pensamiento científico de los estudiantes.

Por otra parte, siguiendo a autores como Popper (1985), se ha sugerido que, por no ser contrastable, la teoría de la evolución es un programa de investigación metafísico más que científico-mecanístico. Por esta razón, si se incorporan explicaciones como la evolutiva dentro del paradigma de construcción del conocimiento de las ciencias 
básicas para Medicina, ello apoyaría más el acercamiento a una visión desde una epistemología constructivista, por oposición a la conductivista. Esto último ya que, según Salas (2002), en un abordaje conductivista solo sería posible incluir el conocimiento verificable, no el metafísico o filosófico.

En el adulto joven, es decir, en el estudiante universitario promedio de la carrera de Medicina, deben consolidarse los procesos mentales que le permitan abandonar el animismo propio del pensamiento infantil, entendiendo por animismo aquella forma de pensar que le atribuye un sentido de propósito a la naturaleza dirigido por una consciencia superior. Según Piaget (1967), no hay ningún experimento o actividad práctica, que lleve al niño a abandonar esta concepción animista, de manera que correspondería a una adecuada visión pedagógica, con un fundamento epistemológico bien definido, facilitar la consolidación de los procesos mentales en las etapas posteriores de la vida (incluida la educación universitaria) de acuerdo con las capacidades cognoscitivas propias del estudiante (Díaz-Barriga et al., 1997).

Ahondando más en el valor educativo de las explicaciones antropomórficas y teleológicas, Talanquer (2007) sugiere que dichas formas de enseñanza en Química (y, por su similitud, se podría también suponer para la enseñanza de Bioquímica) poseen un cierto valor pedagógico: la personificación de los átomos y moléculas ha ayudado a los químicos a visualizar y controlar un mundo que de otro modo sería abstracto y no familiar, y ha ayudado a comunicar este conocimiento a sus colegas y a sus estudiantes. Sin embargo, este autor también señala el evidente peligro que existe cuando las explicaciones teleológicas o similares se ofrecen sin una discusión que las acompañe. Esta discusión debería, tal y como he estado señalando, delimitar los alcances de la explicación, enfatizar que la antropomorfización de un fenómeno bioquímico o fisiológico tiene solamente valor pedagógico y que no implica la existencia de un finalismo por sí mismo. Como parte de la discusión que debe acompañar al uso de las explicaciones teleológicas, antropomórficas o similares, está la contextualización de tales explicaciones dentro del proceso de "transposición didáctica" (explicado ya en la sección 2). Debe recordarse que en la "transposición didáctica" el conocimiento científico establecido se altera o reestructura con propósitos de comunicación o enseñanza. Este tipo de transposiciones del conocimiento son influidas por las epistemologías de los autores de los libros de texto y de los profesores, por las experiencias y filosofías de la enseñanza, por el conocimiento del contenido pedagógico y hasta por interacciones con otras personas que piensan acerca del problema del contenido y los métodos de enseñanza. De manera que, siendo las transposiciones un mecanismo válido, debería realizarse de la manera más consciente y controlada posible, por ejemplo observando cuidadosamente la epistemología subyacente.

En la propuesta que aquí se plantea se considera que es importante crear oportunidades de aprendizaje para que los estudiantes reflexionen sobre el significado real y las implicaciones de las formulaciones teleológicas, evalúen sus ventajas y desventajas comparadas con otros tipos de formulaciones, y trasladen las explicaciones teleológicas a las explicaciones causales (Talanquer, 2007). Esto podría facilitarse procurando un aprendizaje más activo, entendiendo como tal el que se centra en el estudiante más que en el docente (Michael, 2006a). Sin embargo, como los señala Salas (2002), esta pedagogía resulta onerosa por la relación número de alumnos-número de profesores y también demanda docentes altamente capacitados y la disponibilidad de recursos didácticos no tradicionales. Por estas razones, si se buscara adoptar esta fundamentación epistemológica, debería prestarse especial atención al problema que representan los grupos muy numerosos de estudiantes y la preparación de los docentes en la elaboración y uso de tecnologías de información y comunicación. 


\section{Conclusiones}

La definición epistemológica, para los efectos del aprendizaje de las ciencias básicas en la carrera de Medicina y en relación con el diseño curricular, se refiere a la forma en que se concibe el abordaje del problema del conocimiento en las operaciones del aprendizaje. Esta definición debería procurar una coherencia entre "explicaciones de una disciplina" y "explicaciones instruccionales". Ante preguntas limítrofes puede surgir en el aula una aparente contradicción entre posiciones epistemológicas diferentes que en sus manifestaciones extremas conducirían por un lado a explicaciones antropomórficas-teleológicas y por otro a explicaciones causales-mecanísticas. Las primeras constituyen contribuciones que los docentes y los libros de texto hacen a los procesos de aprendizaje y son herramientas poderosas para ayudar a los estudiantes a entender conceptos, ideas, eventos y procedimientos. Poseen un valor heurístico en el abordaje teleológico ya que convierten una larga formulación en una más breve creando empatía hacia los temas científicos.

En la práctica docente de las ciencias básicas (Bioquímica y Fisiología) de la carrera de Medicina de la Universidad de Costa Rica no está permeando explícitamente una fundamentación epistemológica claramente definida. Y aún más, no parece existir conciencia acerca de las implicaciones de esta carencia y de los efectos que la misma produce en los procesos de enseñanza y aprendizaje. Por ello, se propone la necesidad de definir esta justificación epistemológica preguntándose qué es lo que necesitan saber y hacer los estudiantes cuando completen los cursos. Dicho de otra manera ¿cuáles son los conocimientos, habilidades y destrezas terminales requeridos por estos cursos?

Por lo anterior, deben aclararse los alcances y limitaciones de las explicaciones causales-mecanísticas y las antropomórficas-teleológicas así como la validez de la transposición didáctica para procurar un aprendizaje activo. Lo anterior podría alcanzarse por medio de la adopción de un enfoque epistemológico constructivista, multidisciplinario e interdisciplinario, que propicie mayormente el aprendizaje activo y el enfoque deductivo. Se concluye que es posible utilizar tanto explicaciones causalesmecanísticas como antropomórficas-teleológicas siempre y cuando se aclare que las primeras se limitan explicar los fenómenos naturales en términos de causas y efectos y las segundas tienen solamente utilidad pedagógica.

\section{Referencias bibliográficas}

Bazar, K. A., Yun, A. J., y Lee, P. Y. (2005). Starve a fever and feed a cold: Feeding and anorexia may be adaptive behavioral modulators of autonomic and $\mathrm{T}$ helper balance [Privar de alimento a la fiebre y alimentar al resfrío. La alimentación y la anorexia pueden ser moduladores conductuales adaptativos del balance autonómico y de T auxiliares]. Medical Hypotheses, 64(6), 1080-1084.

Díaz-Barriga, F., Lule, M. L, Pacheco, D., Saad, E., y Rojas-Drummond, S. (1997). Metodología de diseño curricular para la educación superior (Reimpresión). México: Trillas.

Ferrater Mora, J. (2007). Diccionario de filosofía abreviado (28a. ed.). Buenos Aires: Sudamericana.

Hernández, A. (1999). Formación pedagógica de los docentes universitarios. Revista Educación, 23 (Especial), 91-104.

Michael, J. (2006a). Where's the evidence that active learning works? [¿Dónde está la evidencia de que el aprendizaje activo funciona?]. Advances in Physiology Education, 30(4), 159-167. 
Michael, J. (2006b). What makes physiology hard for students to learn? Results of a faculty survey [¿Qué hace que la fisiología sea difícil de aprender para los estudiantes. Resultados de una encuesta al personal docente]. Advances in Physiology Education, 31(1), 34-40.

Modell, H. I. (2007). Helping students make sense of physiological mechanisms: The "view from the inside" [Ayudando a los estudiantes a comprender los mecanismos fisiológicos: La "vista desde adentro"]. Advances in Physiology Education, 31(2), 186-192.

Piaget, J. (1967). The child's conception of the world [La concepción del mundo en el niño]. New Jersey: Littlefield, Adams \& Co.

Popper, K. R. (1985). Búsqueda sin término. Una autobiografía intelectual (Reimpresión). Madrid: Tecnos.

Pluhar, W. (2006). AT2-AT3-profiling: A new look at synonymous codon usage [E] perfil de AT2-AT3: Un nuevo vistazo al uso de codones sinónimos]. Journal of Theoretical Biology, 243(3), 308-321.

Quesada, M. E., Cedeño, M. A, y Zamora, J. M. (2001). El diseño curricular en los planes de estudio: Aspectos teóricos y guía metodológica. Heredia, Costa Rica: EUNA.

Richardson, D. (1990). A survey of students' notions of body function as teleologic or mechanistic [Medición de las nociones de los estudiantes acerca de la función del cuerpo como teleológicas o mecanísticas]. Advances in Physiology Education, 258(6), S8-S10.

Roldán, L. (2005). Elementos para evaluar planes de estudio en la educación superior. Revista Educación, 29(1), 111-123.
Salas, F. (2002). Epistemología, educación y tecnología educativa. Revista Educación, 26(1), 9-18.

Talanquer, V. (2007). Explanations and teleology in chemistry education [Explicaciones y teleología en la educación de la química]. International Journal of Science Education, 29(7), 853-870.

Tamir, P., y Zohar, A. (1991). Anthropomorphism and teleology in reasoning about biological phenomena [Antropomorfismo y teleología en el razonamiento acerca de los fenómenos biológicos]. Science Education, 75(1), 57-67.

Universidad de Costa Rica. Escuela de Medicina. (2005). Informe del proceso de autoevaluación para la reacreditación de la carrera de Bachillerato en Ciencias Médicas y Licenciatura en Medicina y Cirugía. Convocatoria 2005. Comisión de Autoevaluación para la Acreditación II. Manuscrito sin publicar.

Yun, A. J., Lee, P. Y., Bazar, K. A., Daniel, S. M., y Doux, J. D. (2005). The incorporation of iodine in thyroid hormone may stem from its role as prehistoric signal of ecologic opportunity: An evolutionary perspective and implications for modern diseases [La incorporación del yodo en las hormonas tiroideas puede provenir de su papel como señal prehistórica de la oportunidad ecológica: Una perspectiva evolutiva e implicaciones para las enfermedades modernas]. $\mathrm{Me}$ dical Hypotheses, 65(4), 804-810.

Zohar, A., y Ginossar, S. (1998). Lifting the taboo regarding teleology and anthropomorphism in biology education-heretical suggestions [Superando el tabú respecto a la teleología y el antropomorfismo en la educación de la biología-sugerencias heréticas]. Science Education, 82(6), 679-697. 